\title{
Very low-grade albuminuria reflects susceptibility to chronic kidney disease in combination with cardiovascular risk factors
}

\author{
Nam Ju Heo ${ }^{1}$, Jeong Myung $\mathrm{Ahn}^{2}$, Tae Woo Lee ${ }^{2,3}$, Ho Jun Chin ${ }^{2,3}$, Ki Young $\mathrm{Na}^{2}$, Dong Wan Chae ${ }^{2}$ \\ and Suhnggwon Kim ${ }^{3,4}$
}

Low-grade albuminuria has been proposed as a cardiovascular risk factor that is below the conventional cut-off point for microalbuminuria, which has been previously identified as a marker for cardiovascular disease and chronic kidney disease (CKD). Metabolic syndrome has also been shown to be related with microalbuminuria and CKD. We assessed the relationship among low-grade albuminuria, CKD and metabolic syndrome among 5998 non-diabetic subjects. The subjects were divided into six groups: subjects with urine albumin-to-creatinine ratio (UACR) $<30 \mathrm{mg} \mathrm{g}^{-1}$ were divided into five groups in accordance with their UACR values, and subjects with $30 \leqslant$ UACR $<300 \mathrm{mg} \mathrm{g}^{-1}$ were allocated to the microalbuminuria group. The prevalence of CKD increased in parallel with increasing UACR values and greater numbers of metabolic syndrome characteristics, which were in turn associated with a reduced UACR cut-off point for an increased prevalence of CKD. Among the subjects with metabolic syndrome, UACR values above $10.2 \mathrm{mgg}^{-1}$ were related to increased CKD prevalence (odds ratio (OR): $2.63,95 \%$ confidence interval (CI) 1.11-6.24), as were values of $30 \mathrm{mg} \mathrm{g}^{-1}$ among those with 1 or 2 components of metabolic syndrome (OR: $2.98,95 \% \mathrm{Cl} 1.83-4.83$ ); elevated UACR was not observed to increase the risk of CKD in subjects who had no components of metabolic syndrome. The cut-off point varied in subjects with various cardiovascular risk profiles such as serum uric acid level, gender or hypertension. Very low levels of albuminuria were associated with increased CKD prevalence. The UACR cut-off point for increased CKD risk varied according to the risk profile, including the number of metabolic syndrome components.

Hypertension Research (2010) 33, 573-578; doi:10.1038/hr.2010.39; published online 9 April 2010

Keywords: chronic kidney disease; metabolic syndrome; microalbuminuria

\section{INTRODUCTION}

Levels of microalbuminuria, which is defined by a urine albumin-tocreatinine ratio (UACR) of $30-300 \mathrm{mg} \mathrm{g}^{-1}$, were originally determined in patients with diabetes mellitus to predict diabetic nephropathy. ${ }^{1}$ It was also demonstrated that microalbuminuria is a risk factor for cardiovascular and kidney diseases in patients with hypertension, as well as in the general population. ${ }^{2-4}$

Recently, the original definition of microalbuminuria has been challenged. Several studies have provided evidence of increased cardiovascular morbidity and mortality when urinary albumin excretion rates are below the currently conventional cut-off point for microalbuminuria, among the general population as well as those with diabetes or hypertension. ${ }^{5-10}$ Although the threshold level at which microalbuminuria is predictive of cardiovascular outcomes is getting lower, there is a paucity of data showing the relationship between low-grade albuminuria and renal risk.

Microalbuminuria has also been considered as a marker of chronic kidney disease (CKD). CKD is a worldwide public health problem. It is a major risk factor for end-stage renal disease and cardiovascular disease, and has been shown to cause premature death. ${ }^{11}$ Nevertheless, little is currently known about the association between low-grade albuminuria and CKD.

Metabolic syndrome is known to be related to microalbuminuria and CKD. ${ }^{12-14}$ The incidence of metabolic syndrome is increasing worldwide and has been associated with an increased risk of mortality from cardiovascular disease.

In this study, we assessed the relationship among low-grade albuminuria, CKD and metabolic syndrome in non-diabetic subjects.

\footnotetext{
${ }^{1}$ Department of Internal Medicine, Healthcare System Gangnam Center, Seoul National University Hospital, Seoul, Republic of Korea; ${ }^{2}$ Department of Internal Medicine, Seoul National University Bundang Hospital, Seongnam, Republic of Korea; ${ }^{3}$ Medical Research Center, Kidney Research Institute, Seoul National University, Seoul, Republic of Korea and ${ }^{4}$ Department of Internal Medicine, Seoul National University Hospital, Seoul, Republic of Korea

Correspondence: Professor HJ Chin, Department of Internal Medicine, Seoul National University Bundang Hospital, 166 Gume-Ro, Bundang-Gu, Seongnam, Gyeonggi-Do 463-707, Republic of Korea.

E-mail: mednep@snubh.org

Received 6 August 2009; revised 20 January 2010; accepted 3 February 2010; published online 9 April 2010
} 


\section{METHODS}

\section{Study population}

This study included a population of Korean adults who had visited the Seoul National University Bundang Hospital for routine health status checkups. This established service program included a medical questionnaire, a physical examination and a series of laboratory tests for a variety of adult diseases. All participants visited the hospital voluntarily, and paid for the service.

A total of 6915 subjects completed a standardized checkup in 2006. Among these, we excluded the following: 523 subjects with diabetes (defined as a history of diabetes mellitus or a fasting blood glucose level of $126 \mathrm{mg}$ per $100 \mathrm{ml}$ or more), 22 subjects with macroalbuminuria (UACR $\geqslant 300 \mathrm{mg} \mathrm{g}^{-1}$ ) and 372 subjects with hematuria (dipstick $\geqslant 1+$ ). A total of 5998 subjects were ultimately included in this study.

\section{Measurements and definitions}

Assessments of medical history, smoking status and medications were based on replies to a standardized questionnaire. Current smokers were defined as those who were habitual smokers at the time of the interview. Blood pressure (BP) was measured using a standardized sphygmomanometer with patients in a sitting position after a 5-min rest, and the average of three measurements was recorded. The height and weight of the subjects were measured with the individuals dressed in only an examination gown and wearing no shoes. Body mass index was expressed as weight in kilograms divided by height in meters $\left(\mathrm{kg} \mathrm{m}^{-2}\right)$. Waist circumference was measured in the standing position at the level of the umbilicus, by a single examiner. Hypertension was defined as systolic BP $\geqslant 140 \mathrm{~mm} \mathrm{Hg}$ or diastolic BP $\geqslant 90 \mathrm{~mm} \mathrm{Hg}$. Participants taking antihypertensive medication were included in this category. Blood samples collected after at least $12 \mathrm{~h}$ of fasting were used for the determination of triglyceride, high-density lipoprotein cholesterol, glucose, creatinine and C-reactive protein (CRP) levels. Serum creatinine concentration was measured using the Jaffe rate reaction. Plasma CRP was measured via particle-enhanced light-scattering immunoassays using a TBA-200FR system (Toshiba, Tokyo, Japan) in accordance with the manufacturer's instructions, and the normal range was set to less than $5 \mathrm{mgl}^{-1}$.

A random single-void urine sample was obtained at the baseline examination to measure the UACR $\left(\mathrm{mgg}^{-1}\right)$ using the clean-catch technique.
The urine sample was obtained after at least $12 \mathrm{~h}$ of fasting. Urinary albumin was measured by an immunoturbidimetric assay, and urinary creatinine was measured using the Jaffe rate reaction. The glomerular filtration rate (GFR) was estimated using the abbreviated equation described in the Modification of Diet in Renal Disease (MDRD) study. ${ }^{11}$ We defined the cut-off points for high serum uric acid as $7 \mathrm{mg}$ per $100 \mathrm{ml}$ in men and $6 \mathrm{mg}$ per $100 \mathrm{ml}$ in women.

Metabolic syndrome was defined in accordance with the criteria of the revised Adult Treatment Panel $\mathrm{III}^{15}$ when a subject exhibited three or more of the known risk factors. Hypertriglyceridemia was defined as $\geqslant 150 \mathrm{mg}$ per $100 \mathrm{ml}$, low high-density lipoprotein cholesterol as $\leqslant 40 \mathrm{mg}$ per $100 \mathrm{ml}$ in men and $\leqslant 50 \mathrm{mg}$ per $100 \mathrm{ml}$ in women; high $\mathrm{BP}$ as a systolic $\mathrm{BP}$ of $\geqslant 130 \mathrm{~mm} \mathrm{Hg}$ or a diastolic BP of $\geqslant 85 \mathrm{~mm} \mathrm{Hg}$, and high fasting glucose was defined as $\geqslant 100 \mathrm{mg}$ per $100 \mathrm{ml}$. Abdominal obesity was defined as a waist circumference of $\geqslant 90 \mathrm{~cm}$ in men and $\geqslant 80 \mathrm{~cm}$ in women, in accordance with the 1998 World Health Organization Asian Pacific Guideline. CKD was defined as a reduced estimated GFR $\left(<60 \mathrm{ml} \mathrm{min}^{-1}\right.$ per $\left.1.73 \mathrm{~m}^{2}\right)$.

\section{Statistical analysis}

The participants were divided into six groups: subjects with low-grade albuminuria (UACR $<30 \mathrm{mg} \mathrm{g}^{-1}$ ) were categorized in quintiles of UACR, and subjects with microalbuminuria $\left(30 \leqslant \mathrm{UACR}<300 \mathrm{mgg}^{-1}\right)$ were defined as the microalbuminuria group. The odds ratios (ORs) for CKD prevalence were calculated for quintiles of UACR and microalbuminuria by logistic regression analysis. All statistical analyses of the data were conducted with SPSS version 12.0 software (SPSS, Chicago, IL, USA). All reported $P$-values were two-tailed, and the threshold of statistical significance was set at $P<0.05$.

\section{RESULTS}

\section{Clinical characteristics of albuminuria groups}

The clinical characteristics of the study population in relationship to albuminuria levels are reported in Table 1 . The distribution of estimated GFR is shown in Figure 1. Overall, 678 subjects (11.3\%) had CKD and 629 subjects $(10.5 \%)$ had metabolic syndrome. The prevalence of CKD increased with UACR across groups (9.1\% in Q1, 10.7\% in Q3, 12.3\% in Q5 and $18.4 \%$ in the microalbuminuria group).

Table 1 Clinical characteristics of albuminuria groups

UACR quintile groups (below microalbuminuria)

\begin{tabular}{|c|c|c|c|c|c|c|c|}
\hline Charateristic & $Q 1(<4.1)$ & Q2 (4.1-5.3) & Q3 (5.4-6.9) & Q4 (7.0-10.1) & Q5 (10.2-29.9) & Microalbuminuria & $\begin{array}{l}\text { P-value by } \\
\text { one-way ANOVA }\end{array}$ \\
\hline Number & 1172 & 1166 & 1125 & 1152 & 1138 & 245 & \\
\hline UACR (IQR) (mgg-1) & $3.30(2.80-4.80)$ & $4.70(4.40-5.00)$ & $6.00(5.60-6.40)$ & $8.20(7.45-8.95)$ & $14.10(10.80-17.40)$ & $48.30(29.35-67.25)$ & $<0.001$ \\
\hline Age (year) & $47.54 \pm 9.6$ & $49.06 \pm 9.78$ & $50.14 \pm 9.99$ & $50.91 \pm 10.43$ & $52.45 \pm 10.46$ & $54.95 \pm 11.57$ & $<0.001$ \\
\hline $\mathrm{CRP}\left(\mathrm{mg} \mathrm{I}^{-1}\right)$ & $1.08 \pm 2.53$ & $1.01 \pm 2.73$ & $1.01 \pm 2.39$ & $1.27 \pm 4.12$ & $1.85 \pm 8.07$ & $2.51 \pm 5.22$ & $<0.001$ \\
\hline $\mathrm{BMI}\left(\mathrm{kg} \mathrm{m}^{-2}\right)$ & $23.78 \pm 2.62$ & $23.58 \pm 2.65$ & $23.44 \pm 2.89$ & $23.67 \pm 3.08$ & $23.91 \pm 3.13$ & $24.95 \pm 3.55$ & $<0.001$ \\
\hline $\mathrm{SBP}(\mathrm{mm} \mathrm{Hg})$ & $112.33 \pm 12.49$ & $113.67 \pm 13.36$ & $113.69 \pm 14.07$ & $115.21 \pm 14.47$ & $118.49 \pm 15.54$ & $124.08 \pm 17.61$ & $<0.001$ \\
\hline $\mathrm{DBP}(\mathrm{mm} \mathrm{Hg})$ & $71.75 \pm 10.57$ & $72.75 \pm 11.13$ & $72.2 \pm 11.52$ & $73.11 \pm 11.71$ & $74.99 \pm 12.44$ & $78.86 \pm 12.44$ & $<0.001$ \\
\hline Estimated GFR & $71.31 \pm 9.29$ & $71.2 \pm 9.94$ & $70.84 \pm 9.26$ & $70.98 \pm 10.06$ & $71.23 \pm 11.13$ & $68.73 \pm 10.72$ & 0.010 \\
\hline FBS (mg per $100 \mathrm{ml}$ ) & $89.29 \pm 10.9$ & $90.05 \pm 10.96$ & $90.19 \pm 11.07$ & $90.81 \pm 11.61$ & $91.94 \pm 12.07$ & $95.09 \pm 11.83$ & $<0.001$ \\
\hline Uric acid (mg per $100 \mathrm{ml}$ ) & $5.92 \pm 1.37$ & $5.56 \pm 1.38$ & $5.37 \pm 1.43$ & $5.38 \pm 1.51$ & $5.52 \pm 1.54$ & $6.1 \pm 1.68$ & $<0.001$ \\
\hline Total cholesterol $\left(\mathrm{mmol} \mathrm{I}^{-1}\right)$ & $199.64 \pm 32.62$ & $200.19 \pm 32.81$ & $203.96 \pm 35.23$ & $205.84 \pm 36.14$ & $208.07 \pm 35.42$ & $208.22 \pm 36.37$ & $<0.001$ \\
\hline LDL (mg per $100 \mathrm{ml}$ ) & $103.37 \pm 24.03$ & $102.7 \pm 24.33$ & $105.08 \pm 25.57$ & $106.56 \pm 26.3$ & $108.14 \pm 26.19$ & $106.82 \pm 26.51$ & $<0.001$ \\
\hline $\mathrm{HDL}$ (mg per $100 \mathrm{ml}$ ) & $56.83 \pm 13.56$ & $59.26 \pm 15.09$ & $60.07 \pm 14.97$ & $59.13 \pm 14.19$ & $58.84 \pm 14.76$ & $57.09 \pm 14.95$ & $<0.001$ \\
\hline Triglyceride (mg per $100 \mathrm{ml}$ ) & $124.31 \pm 72.91$ & $118.09 \pm 71.02$ & $118.57 \pm 76.78$ & $125.18 \pm 79.37$ & $129.44 \pm 89.23$ & $150.3 \pm 126.64$ & $<0.001$ \\
\hline Men $(\%)$ & 75.0 & 60.8 & 47.2 & 46.0 & 50.8 & 62.4 & \\
\hline Smoking (\%) & 67.9 & 56.9 & 48.7 & 48.1 & 50.5 & 60.9 & \\
\hline HT drug (\%) & 8.2 & 10.4 & 12.9 & 14.9 & 19.4 & 39.6 & \\
\hline Chronic kidney disease (\%) & 9.1 & 11.2 & 10.7 & 11.5 & 12.3 & 18.4 & \\
\hline
\end{tabular}

Abbreviations: ANOVA, analysis of variance; BMI, body mass index; CRP, C-reactive protein; DBP, diastolic blood pressure; FBS, fasting blood sugar; GFR, glomerular filtration rate;

$\mathrm{HDL}$, high-density lipoprotein; IQR, interquartile range; LDL, low-density lipoprotein; SBP, systolic blood pressure; UACR, urine albumin-to-creatinine ratio; HT drug, percent of the individuals taking antihypertensive drugs. 
Association of UACR with the prevalence of CKD

Among all subjects, UACR levels of over $10.5 \mathrm{mg} \mathrm{g}^{-1}$ were associated with increased CKD prevalence. In the multivariate model, the OR for CKD increased with UACR value. Low-grade albuminuria (UACR, $10.1-29.9 \mathrm{mg} \mathrm{g}^{-1}$; OR, 1.37; 95\% confidence interval (CI) 1.05-1.78) was associated with increased CKD prevalence as well as microalbuminuria (30.0-290.69 $\mathrm{mg} \mathrm{g}^{-1}$; OR, 2.19; 95\% CI 1.50-3.20) (Table 2).

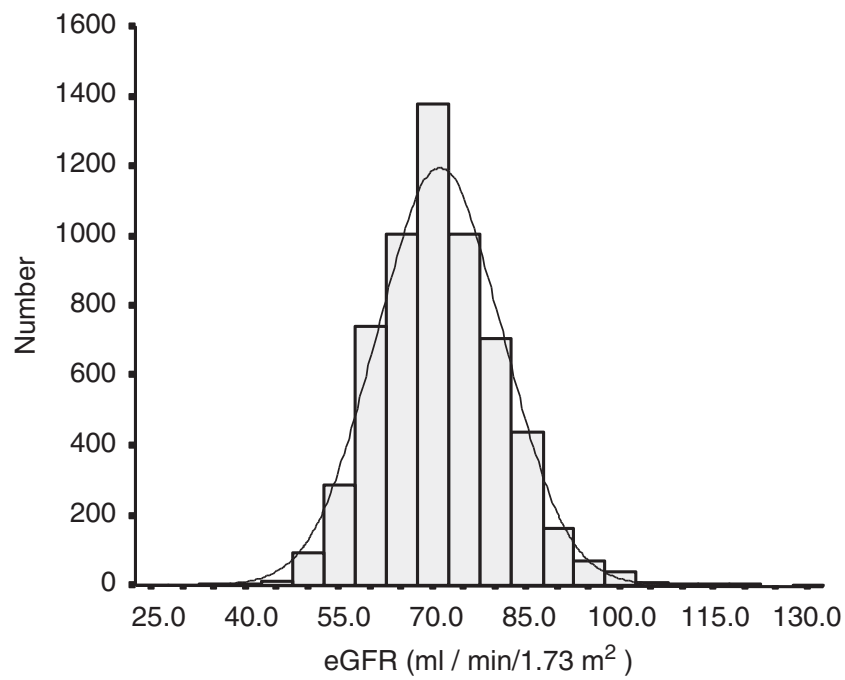

Figure 1 The distribution of estimated glomerular filtration rate (eGFR).

Table 2 Odds ratios for chronic kidney disease according to UACR levels

\begin{tabular}{|c|c|c|c|}
\hline & Number & $\begin{array}{c}\text { Multivariate-adjusted } \\
\text { OR }^{\mathrm{a}}(95 \% \mathrm{Cl})\end{array}$ & P-value \\
\hline \multicolumn{4}{|c|}{ UACR quintile groups (below microalbuminuria) } \\
\hline Q1 $\left(\right.$ UACR $\left.<4.1 \mathrm{mgg}^{-1}\right)$ & 1172 & Ref. & \\
\hline Q2 (4.1-5.3) & 1166 & $1.23(0.94-1.61)$ & 0.129 \\
\hline Q3 (5.4-6.9) & 1125 & $1.16(0.88-1.53)$ & 0.281 \\
\hline Q4 (7.0-10.1) & 1152 & $1.27(0.97-1.66)$ & 0.078 \\
\hline Q5 (10.2-29.9) & 1138 & $1.37(1.05-1.78)$ & 0.021 \\
\hline Microalbuminuria & 245 & $2.19(1.50-3.20)$ & $<0.001$ \\
\hline
\end{tabular}

Association of UACR with the prevalence of CKD is dependent on the number of metabolic syndrome components present The prevalence of CKD increased with the number of metabolic syndrome components $(10.5 \%$ in subjects with no or one metabolic syndrome component, $13.1 \%$ in patients with two components and $13.7 \%$ in those with metabolic syndrome). In the multivariate model, higher metabolic component numbers were associated with increased CKD prevalence. Relative to subjects with 0 or 1 component(s) of metabolic syndrome, those with 2 components of metabolic syndrome (still not sufficient for a diagnosis of metabolic syndrome) had ORs for CKD of 1.28 (95\% CI 1.04-1.56), and participants with metabolic syndrome had ORs of 1.33 (95\% CI 1.03-1.71); these results were independent of age, sex, hypertension, smoking status, body mass index, serum low-density lipoprotein, uric acid and CRP levels.

The UACR cut-off point for an increased prevalence for CKD decreased progressively with a higher number of metabolic syndrome components (Table 3). Among individuals without components of metabolic syndrome, elevated UACR levels did not increase the ORs for $\mathrm{CKD}$ even with regard to the level of microalbuminuria. Among those with 1 or 2 metabolic syndrome components, only microalbuminuria was associated with an increased OR for CKD (30.0$290.69 \mathrm{mg} \mathrm{g}^{-1}$; OR: $2.98,95 \%$ CI 1.83-4.83). CKD prevalence was elevated in the highest quintile $\left(10.2-29.9 \mathrm{mg} \mathrm{g}^{-1}\right.$; OR: 2.63 ; $95 \%$ CI 1.11-6.24) of subjects with metabolic syndrome.

Association of UACR with the prevalence of CKD is dependent on serum uric acid level, gender and hypertension

When classified according to serum uric acid levels, the UACR cut-off value for increased prevalence of CKD risk was $7.0 \mathrm{mg} \mathrm{g}^{-1}$ in subjects with high uric acid levels. In the multivariate model, $\mathrm{CKD}$ prevalence was elevated in the fourth quintile $\left(7.0-10.1 \mathrm{mg} \mathrm{g}^{-1}\right.$; OR: $1.60,95 \% \mathrm{CI}$ 1.05-2.45) among those with high uric acid levels, whereas there was no significant increase in OR with UACR among those with low uric acid levels (Table 4). The OR for CKD increased in parallel with UACR group: the OR for CKD was 1.86 in the highest quintile (10.2$29.9 \mathrm{mg} \mathrm{g}^{-1}$; 95\% CI 1.25-2.77) and the OR was 2.42 in the microalbuminuria group (30.0-290.69 $\mathrm{mg} \mathrm{g}^{-1}$; 95\% CI 1.39-4.19). Likewise, in the analysis classified by gender, the multivariate-adjusted OR for CKD was elevated in the highest quintile $\left(10.2-29.9 \mathrm{mg} \mathrm{g}^{-1}\right.$ OR: 1.42 , 95\% CI 0.99-1.98) among men, although this difference was statistically insignificant. Among women, the OR was elevated only in the microalbuminuria group $\left(30.0-290.69 \mathrm{mgg}^{-1}\right.$; OR: 1.94 ; 95\% CI 1.04-3.65) (Table 5). Among individuals with hypertension,

Table 3 Odds ratios for chronic kidney disease according to the number of metabolic syndrome components and UACR levels

\begin{tabular}{|c|c|c|c|c|c|c|c|c|c|}
\hline & \multicolumn{3}{|c|}{ Component $=0$} & \multicolumn{3}{|c|}{ Components $=1$ or 2} & \multicolumn{3}{|c|}{ Components $=3,4$ or 5} \\
\hline & \multicolumn{3}{|c|}{ Multivariate-adjusted } & \multicolumn{3}{|c|}{ Multivariate-adjusted } & \multicolumn{3}{|c|}{ Multivariate-adjusted } \\
\hline & Number & $O R^{a}(95 \% C l)$ & P-value & Number & $O R^{a}(95 \% C l)$ & P-value & Number & $O R^{a}(95 \%$ Cl) & P-value \\
\hline \multicolumn{10}{|c|}{ UACR quintile groups (below microalbuminuria) } \\
\hline Q1 (UACR < $\left.4.1 \mathrm{mgg}^{-1}\right)$ & 527 & Ref. & & 565 & Ref. & & 78 & Ref. & \\
\hline Q2 (4.1-5.3) & 490 & $0.94(0.62-1.43)$ & 0.788 & 589 & $1.45(0.99-2.13)$ & 0.053 & 86 & $1.34(0.48-3.70)$ & 0.579 \\
\hline Q3 (5.4-6.9) & 457 & $0.95(0.62-1.46)$ & 0.826 & 577 & $1.19(0.80-1.77)$ & 0.381 & 90 & $1.56(0.58-4.18)$ & 0.376 \\
\hline Q4 (7.0-10.1) & 440 & $1.24(0.82-1.87)$ & 0.313 & 557 & $1.29(0.87-1.91)$ & 0.207 & 152 & $1.03(0.40-2.66)$ & 0.953 \\
\hline Q5 (10.2-29.9) & 349 & $0.92(0.58-1.46)$ & 0.719 & 622 & $1.32(0.90-1.94)$ & 0.148 & 165 & $2.63(1.11-6.24)$ & 0.028 \\
\hline Microalbuminuria & 40 & $0.66(0.19-2.22)$ & 0.497 & 147 & $2.98(1.83-4.83)$ & $<001$ & 58 & $1.86(0.65-5.34)$ & 0.247 \\
\hline
\end{tabular}

Abbreviations: BMI, body mass index; Cl, confidence interval; CRP, C-reactive protein; LDL, low-density lipoprotein; OR, odds ratio; UACR, urine albumin-to-creatinine ratio.

aAdjusted for age, gender, smoking, BMI, serum LDL level, serum uric acid level and serum CRP level (log transformed). 
Table 4 Odds ratios for chronic kidney disease according to uric acid and UACR levels

\begin{tabular}{|c|c|c|c|c|c|c|}
\hline & \multicolumn{3}{|c|}{ Low } & \multicolumn{3}{|c|}{ High } \\
\hline & \multicolumn{3}{|c|}{ Multivariate-adjusted } & \multicolumn{3}{|c|}{ Multivariate-adjusted } \\
\hline & Number & $O R^{a}(95 \% C l)$ & P-value & Number & $O R^{a}(95 \% C l)$ & P-value \\
\hline \multicolumn{7}{|c|}{ UACR quintile groups (below microalbuminuria) } \\
\hline Q1 $\left(\right.$ UACR $\left.<4.1 \mathrm{mgg}^{-1}\right)$ & 593 & Ref. & & 579 & Ref. & \\
\hline Q2 (4.1-5.3) & 701 & $1.11(0.78-1.58)$ & 0.550 & 465 & $1.26(0.83-1.92)$ & 0.277 \\
\hline Q5 (10.2-29.9) & 707 & $0.91(0.63-1.32)$ & 0.616 & 431 & $1.86(1.25-2.77)$ & 0.002 \\
\hline Microalbuminuria & 125 & $1.66(0.97-2.86)$ & 0.077 & 120 & $2.42(1.39-4.19)$ & 0.002 \\
\hline
\end{tabular}

Abbreviations: BMI, body mass index; $\mathrm{Cl}$, confidence interval; CRP, C-reactive protein; LDL, low-density lipoprotein; OR, odds ratio; UACR, urine albumin-to-creatinine ratio.

${ }^{a}$ Adjusted for age, gender, hypertension, smoking, BMI, serum LDL level and serum CRP level (log transformed).

Table 5 Odds ratios (ORs) for chronic kidney disease according to gender and UACR levels

\begin{tabular}{|c|c|c|c|c|c|c|}
\hline & \multicolumn{3}{|c|}{ Female } & \multicolumn{3}{|c|}{ Male } \\
\hline & \multicolumn{3}{|c|}{ Multivariate-adjusted } & \multicolumn{3}{|c|}{ Multivariate-adjusted } \\
\hline & Number & $O R^{\mathrm{a}}(95 \% \mathrm{Cl})$ & P-value & Number & $O R^{a}(95 \% C l)$ & P-value \\
\hline \multicolumn{7}{|c|}{ UACR quintile groups (below microalbuminuria) } \\
\hline Q1 $\left(\right.$ UACR $\left.<4.1 \mathrm{mgg}^{-1}\right)$ & 293 & Ref. & & 876 & Ref. & \\
\hline Q2 (4.1-5.3) & 457 & $1.12(0.71-1.77)$ & 0.616 & 709 & $1.22(0.87-1.72)$ & 0.240 \\
\hline Q5 (10.2-29.9) & 559 & $1.03(0.65-1.61)$ & 0.914 & 577 & $1.40(0.99-1.98)$ & 0.060 \\
\hline Microalbuminuria & 92 & $1.94(1.04-3.65)$ & 0.039 & 153 & $1.76(1.06-2.94)$ & 0.029 \\
\hline
\end{tabular}

Abbreviations: BMI, body mass index; CI, confidence interval; CRP, C-reactive protein; LDL, low-density lipoprotein; OR, odds ratio; UACR, urine albumin-to-creatinine ratio.

aAdjusted for age, hypertension, smoking, BMI, serum uric acid level, serum LDL level and serum CRP level (log transformed).

Table 6 Odds ratios for chronic kidney disease according to the presence or absence of hypertension and UACR levels

\begin{tabular}{|c|c|c|c|c|c|c|}
\hline & \multicolumn{3}{|c|}{ Multivariate-adjusted } & \multicolumn{3}{|c|}{ Multivariate-adjusted } \\
\hline & Number & $O R^{a}(95 \% C l)$ & P-value & Number & $O R^{a}(95 \% C l)$ & P-value \\
\hline \multicolumn{7}{|c|}{ UACR quintile groups (below microalbuminuria) } \\
\hline Q1 $\left(\right.$ UACR $\left.<4.1 \mathrm{mgg}^{-1}\right)$ & 1019 & Ref. & & 152 & Ref. & \\
\hline Q2 (4.1-5.3) & 986 & $1.15(0.86-1.54)$ & 0.343 & 180 & $1.64(0.76-3.54)$ & 0.211 \\
\hline Q5 (10.2-29.9) & 793 & $1.12(0.82-1.53)$ & 0.479 & 344 & $1.98(0.99-3.97)$ & 0.053 \\
\hline Microalbuminuria & 113 & $1.44(0.81-2.56)$ & 0.210 & 0.196 & $3.02(1.42-6.43)$ & 0.004 \\
\hline
\end{tabular}

Abbreviations: BMI, body mass index; CI, confidence interval; CRP, C-reactive protein; LDL, low-density lipoprotein; OR, odds ratio; UACR, urine albumin-to-creatinine ratio.

${ }^{a}$ Adjusted for age, gender, smoking, BMI, serum uric acid level, serum LDL level and serum CRP level (log transformed).

the OR for CKD was elevated in the microalbuminuria group (30.0-290.69 $\mathrm{mgg}^{-1}$; OR: 3.02; 95\% CI 1.42-6.43), whereas no significant increase in OR according to UACR was noted in subjects who did not have hypertension (Table 6). A slightly stronger tendency was noted according to gender in the hypertensive subjects. Among hypertensive men, the OR of CKD increased by 3.37-fold in the microalbuminuria group $\left(30.0-290.69 \mathrm{mg} \mathrm{g}^{-1} ; 95 \%\right.$ CI $\left.1.34-8.28\right)$, whereas no significant increase was observed in women.

\section{DISCUSSION}

The principal objective of this study was to investigate the relationship among low-grade albuminuria, metabolic syndrome and CKD in nondiabetic subjects. The results of this study show that even low levels of albuminuria are associated with increased CKD prevalence, particularly, in populations with high cardiovascular risk profiles, including metabolic syndrome. Moreover, our large cross-sectional analysis shows that a large number of metabolic syndrome components are 
associated with increased CKD prevalence, as well as a reduced UACR cut-off point for increased CKD prevalence. Thus, low-grade albuminuria might have clinical implications for the prediction of CKD, particularly, in the populations with more metabolic syndrome components, independent of diabetes.

These data are in line with recent studies suggesting that the relationship between albuminuria and cardiovascular risk is not restricted to the microalbuminuria range, and extends to very lowgrade albuminuria. Several studies provide evidence of increased cardiovascular morbidity and mortality at urinary albumin excretion rates below the conventional cut-off point for microalbuminuria in the general population, in addition to patients with diabetes or hypertension. ${ }^{5-10}$ Klausen et al. ${ }^{5}$ found that a UACR value above the upper quartile, that is, $4.8 \mathrm{mcg} \mathrm{min}^{-1}$, is associated with an increased risk of coronary heart disease (relative risk 2.0; 95\% CI 1.4-3.0) and death (relative risk 1.9; 95\% CI 1.5-2.4), independent of age, sex, renal creatinine clearance, diabetes mellitus, hypertension, and plasma lipids in non-diabetic and non-hypertensive adults. In the MONICA/KORA substudy, ${ }^{10}$ it was noted that UACR levels of $4-10 \mathrm{mg} \mathrm{g}^{-1}$ may be associated with increased cardiovascular risk, considering the results of several studies, including the Framingham Heart Study, even though this level is far below the currently used threshold of $30 \mathrm{mg} \mathrm{g}^{-1}$ UACR. In our study, we demonstrated that low albuminuria levels were associated with increased CKD prevalence, particularly, in the populations with high-risk cardiovascular profiles, such as metabolic syndrome. The UACR cut-off point in our study $\left(10.2 \mathrm{mgg}^{-1}\right)$ for increased CKD prevalence was similar to those suggested in a previous study for cardiovascular risk. ${ }^{10}$ These results support the notion that low-grade albuminuria may be associated with both cardiovascular and renal risks such as microalbuminuria.

In this study, it was found that the presence of more metabolic syndrome components is associated with increased CKD prevalence and a decreased UACR cut-off point for CKD. In subjects exhibiting no components of metabolic syndrome, elevated UACR did not increase CKD risk, even in the microalbuminuria group. The UACR cut-off point for CKD was $30 \mathrm{mg} \mathrm{g}^{-1}$ in those with 1 or 2 components of metabolic syndrome, and the cut-off point was $10.2 \mathrm{mg} \mathrm{g}^{-1}$ in the subjects with metabolic syndrome. This means that microalbuminuria may be clinically significant in subjects with only 1 or 2 components of metabolic syndrome, and a UACR value of even $10.2 \mathrm{mg} \mathrm{g}^{-1}$ may be worth noting in those subjects with metabolic syndrome. We also analyzed the combined effect of low-grade albuminuria and the accumulation of eight cardiovascular risk profiles including lowdensity lipoprotein, uric acid and CRP on the prevalence of CKD. The results were similar to those shown in the analyses that investigated five components of metabolic syndrome, although the UACR cut-off point for CKD was $30 \mathrm{mgg}^{-1}$ in those with two or three risk factors.

The mechanisms that underlie the observed link between low-grade albuminuria and CKD may involve insulin resistance, as suggested by our findings that metabolic syndrome components influence the UACR cut-off point for CKD. Recently, microalbuminuria has been identified as an indicator of insulin resistance and of increased renal and cardiovascular risks (also known as cardio-renal syndrome) associated with metabolic syndrome. ${ }^{16,17}$ Insulin resistance has been reported to increase the risk of CKD. ${ }^{18,19}$ Chen et al. ${ }^{18,19}$ determined that metabolic syndrome ${ }^{19}$ and insulin resistance estimated by a homeostasis model assessment ${ }^{18}$ were associated with an increased CKD risk in participants in the NHANES III study.

Among subjects with high serum uric acid, a UACR above $7.0 \mathrm{mg} \mathrm{g}^{-1}$ was related to an increased CKD prevalence in our study, whereas we noted no significant increase in ORs for CKD according to UACR in those subjects with low uric acid levels. This is lower than the level that has been detected among subjects with metabolic syndrome or hypertension. Uric acid has been related to cardiovascular morbidity and mortality in a number of previous studies. ${ }^{20,21}$ In the MONICA cohort, hyperuricemia was associated significantly with cardiovascular mortality, independent of body mass index, hypertension, diuretic or alcohol use and smoking. ${ }^{22}$ Uric acid has also been identified as an independent predictor of microalbuminuria in healthy subjects. ${ }^{23}$ Consistent with these studies, elevated serum uric acid levels affected the prevalence of CKD and the cut-off point for CKD in our study. Further studies will be required to determine the relevant mechanisms involved in this process.

Similar results were observed in this study, supporting the notion that the CKD cut-off point varies in subjects with various cardiovascular risk profiles. Consistent with the results of previous studies, the risk for CKD according to UACR differs between men and women. Data from the Framingham Heat Study ${ }^{8}$ shows that the cut-off for an elevated rate of cardiovascular events was a UACR of $3.9 \mathrm{mgg}^{-1}$ for men and a UACR of $7.5 \mathrm{mgg}^{-1}$ for women. The gender-specific differences in cut-off points may be due to different urinary creatinine excretion levels. A second explanation for this difference might be found in the fact that women are generally considered to have lowerrisk cardiovascular profiles than men. Likewise, the cut-off for elevated CKD prevalence varies according to the presence or absence of hypertension, and the accumulation of eight cardiovascular risk profiles including low-density lipoprotein, uric acid and CRP not only the components of metabolic syndrome (Supplementary Tables 1,2). Thus, low-grade albuminuria may have a variable clinical significance according to the risk profiles of the subjects, independent of diabetes.

As metabolic syndrome is associated with increased CKD risk, the number of components affects the risk of CKD. ${ }^{19,24}$ In one study, the risk for $\mathrm{CKD}$ was reported to increase progressively with higher numbers of metabolic syndrome components. ${ }^{19}$ Compared with participants with 0 or 1 components of metabolic syndrome, participants with 2, 3, 4 and 5 metabolic syndrome components had multivariate-adjusted ORs of 2.21 (CI 1.16-4.24), 3.38 (CI 1.487.69), 4.23 (CI 2.06-8.63) and 5.85 (CI 3.11-11.0), respectively. Consistent with these results, higher numbers of metabolic syndrome components were found to be associated with increased CKD prevalence in our study. Although a similar tendency was noted, the ORs observed in our study were lower. This difference may be caused by the exclusion of subjects with diabetes or macroalbuminuria; nondiabetic subjects with lower UACR values are expected to exhibit CKD prevalence that is lower than that of the general population. Moreover, metabolic syndrome is known to be less prevalent in Asian populations than in Western populations. In a study conducted with Asian, non-diabetic and non-hypertensive subjects, ${ }^{24}$ the ORs for CKD as related to the number of metabolic syndrome components were lower than those observed in a previous study that analyzed a Western population.

Among subjects with metabolic syndrome, the prevalence of CKD was not significantly elevated in accordance with microalbuminuria levels, whereas the prevalence of $\mathrm{CKD}$ was elevated in the highest quintile. This unexpected result may be the consequence of the inclusion of a relatively small number of subjects with metabolic syndrome and microalbuminuria. As we excluded those subjects with diabetes or macroalbuminuria, the number of patients with metabolic syndrome, CKD and microalbuminuria was only nine. Further studies with a larger sample size will be required. 
Several limitations of our study should be noted. First, our study was a cross-sectional study and the cohort was voluntary. The study design may have induced some bias, and may also have made it difficult to infer causality between low-grade albuminuria and CKD risk. A prospective study will be necessary to elucidate the mechanism underlying the relationship between low-grade albuminuria and CKD. Second, urinary albumin excretion was assessed in only one morning urine sample. Although timed (for example, 24-h or overnight) urine collections were originally used to measure albumin excretion, good correlations were reported between the UACR and albuminuria levels measured in the overnight and 24-h urine collections. However, it has been recommended that several samples should be obtained, because of the variability and false-positive results that plague microalbuminuria measurements. ${ }^{25}$ To prevent the possibility that contamination may result in false-positive data, we excluded subjects with hematuria from our analysis. Another limitation is that GFR was not measured in the 24-h urine collection, but was estimated with the MDRD formula. Although the MDRD formula has been used extensively in large epidemiological studies, as well as in clinical practice, it is yet to be validated for the Korean population. The median GFR in our subjects was $71 \mathrm{ml} \mathrm{min}^{-1}$ per $1.73 \mathrm{~m}^{2}$, which was similar to that reported in a study of Japanese subjects, ${ }^{26}$ but lower than that reported in other studies of general US and Chinese populations. ${ }^{14,19,27}$ One possible explanation may be that the equation underestimates GFR in Koreans; alternatively, Koreans may be prone to renal damage. Validation of the MDRD formula for the Korean population is another necessary step that will require additional research.

In conclusion, we determined that very low levels of albuminuria were associated with increased CKD prevalence, and that the UACR cut-off point for increased CKD risk varied according to a subject's risk profile, most notably the number of metabolic syndrome components. Thus, in populations with high cardiovascular risk, therapeutic interventions targeted at modifying renal and cardiovascular risk factors that may be of greatest benefit to those who have UACR values below the current conventional cut-off point for microalbuminuria.

1 Mogensen CE, Chachati A, Christensen CK, Close CF, Deckert T, Hommel E, Kastrup J, Lefebvre P, Mathiesen ER, Feldt-Rasmussen B. Microalbuminuria: an early marker of renal involvement in diabetes. Uremia Invest 1985; 9: 85-95.

2 Ruggenenti P, Perna A, Gherardi G, Garini G, Zoccali C, Salvadori M, Scolari F, Schena FP, Remuzzi G. Renoprotective properties of ACE-inhibition in non-diabetic nephropathies with non-nephrotic proteinuria. Lancet 1999; 354: 359-364.

3 Stuveling EM, Hillege HL, Bakker SJ, Asselbergs FW, de Jong PE, Gans RO, de Zeeuw D. C-reactive protein and microalbuminuria differ in their associations with various domains of vascular disease. Atherosclerosis 2004; 172: 107-114.

4 Verhave JC, Gansevoort RT, Hillege HL, Bakker SJ, De Zeeuw D, de Jong PE. An elevated urinary albumin excretion predicts de novo development of renal function impairment in the general population. Kidney Int Supp/ 2004; 92: S18-S21.

5 Klausen K, Borch-Johnsen K, Feldt-Rasmussen B, Jensen G, Clausen P, Scharling H, Appleyard M, Jensen JS. Very low levels of microalbuminuria are associated with increased risk of coronary heart disease and death independently of renal function, hypertension, and diabetes. Circulation 2004; 110: 32-35.
6 Hillege HL, Fidler V, Diercks GF, van Gilst WH, de Zeeuw D, van Veldhuisen DJ, Gans $\mathrm{RO}$, Janssen WM, Grobbee DE, de Jong PE. Urinary albumin excretion predicts cardiovascular and noncardiovascular mortality in general population. Circulation 2002; 106: 1777-1782.

7 Kramer H, Jacobs Jr DR, Bild D, Post W, Saad MF, Detrano R, Tracy R, Cooper R, Liu K. Urine albumin excretion and subclinical cardiovascular disease. The Multi-Ethnic Study of Atherosclerosis. Hypertension 2005; 46: 38-43.

8 Arnlov J, Evans JC, Meigs JB, Wang TJ, Fox CS, Levy D, Benjamin EJ, D'Agostino RB, Vasan RS. Low-grade albuminuria and incidence of cardiovascular disease events in nonhypertensive and nondiabetic individuals: the Framingham Heart Study. Circulation 2005; 112: 969-975.

9 Wang TJ, Evans JC, Meigs JB, Rifai N, Fox CS, D'Agostino RB, Levy D, Vasan RS. Lowgrade albuminuria and the risks of hypertension and blood pressure progression. Circulation 2005; 111: 1370-1376.

10 Lieb W, Mayer B, Stritzke J, Doering A, Hense HW, Loewel H, Erdmann J, Schunkert H. Association of low-grade urinary albumin excretion with left ventricular hypertrophy in the general population: the MONICA/KORA Augsburg Echocardiograph-ic Substudy. Nephrol Dial Transplant 2006; 21: 2780-2787.

11 National Kidney Foundation. K/DOQI clinical practice guidelines for chronic kidney disease: evaluation, classification, and stratification. Am J Kidney Dis 2002; 39: S1-S266.

12 Choi HS, Ryu SH, Lee KB. The relationship of microalbuminuria with metabolic syndrome. Nephron Clin Pract 2006; 104: c85-c93.

13 Palaniappan L, Carnethon M, Fortmann SP. Association between microalbuminuria and the metabolic syndrome: NHANES III. Am J Hypertens 2003; 16: 952-958.

14 Kurella M, Lo JC, Chertow GM. Metabolic syndrome and the risk for chronic kidney disease among nondiabetic adults. J Am Soc Nephrol 2005; 16 : 2134-2140.

15 Expert Panel on Detection, Evaluation, and Treatment of High Blood Cholesterol in Adults. Executive Summary of The Third Report of The National Cholesterol Education Program (NCEP) Expert Panel on Detection, Evaluation, And Treatment of High Blood Cholesterol In Adults (Adult Treatment Panel III). JAMA 2001; 285: 2486-2497.

16 Ruggenenti P, Remuzzi G. Time to abandon microalbuminuria? Kidney Int 2006; 70: $1214-1222$.

17 Ninomiya T, Kiyohara Y. Albuminuria and chronic kidney disease in association with the metabolic syndrome. J Cardiometab Syndr 2007; 2: 104-107.

18 Chen J, Muntner P, Hamm LL, Fonseca V, Batuman V, Whelton PK, He J. Insulin resistance and risk of chronic kidney disease in nondiabetic US adults. J Am Soc Nephrol 2003; 14: 469-477.

19 Chen J, Muntner P, Hamm LL, Jones DW, Batuman V, Fonseca V, Whelton PK, He J. The metabolic syndrome and chronic kidney disease in U.S. adults. Ann Intern Med 2004; 140: $167-174$.

20 Tseng $\mathrm{CH}$. Correlation of uric acid and urinary albumin excretion rate in patients with type 2 diabetes mellitus in Taiwan. Kidney Int 2005; 68: 796-801.

21 Johnson RJ, Segal MS, Srinivas T, Ejaz A, Mu W, Roncal C, Sanchez-Lozada LG, Gersch M, Rodriguez-Iturbe B, Kang DH, Acosta JH. Essential hypertension, progressive renal disease, and uric acid: a pathogenetic link? J Am Soc Nephrol 2005; 16: 1909-1919.

22 Liese AD, Hense HW, Lowel H, Doring A, Tietze M, Keil U. Association of serum uric acid with all-cause and cardiovascular disease mortality and incident myocardial infarction in the MONICA Augsburg cohort. World Health Organization Monitoring Trends and Determinants in Cardiovascular Diseases. Epidemiology 1999; 10: 391-397.

23 Bellomo G, Berardi P, Saronio P, Verdura C, Esposito A, Laureti A, Venanzi S, Timio F, Timio M. Microalbuminuria and uric acid in healthy subjects. J Nephrol 2006; 19: 458-464.

24 Lee JE, Choi SY, Huh W, Kim YG, Kim DJ, Oh HY. Metabolic syndrome, C-reactive protein, and chronic kidney disease in nondiabetic, nonhypertensive adults. Am J Hypertens 2007; 20: 1189-1194.

25 Molitch ME, DeFronzo RA, Franz MJ, Keane WF, Mogensen CE, Parving HH. Diabetic nephropathy. Diabetes Care 2003; 26(Suppl 1): S94-S98.

26 Ninomiya T, Kiyohara Y, Kubo M, Yonemoto K, Tanizaki Y, Doi Y, Hirakata H, lida M. Metabolic syndrome and CKD in a general Japanese population: the Hisayama Study. Am J Kidney Dis 2006; 48: 383-391.

27 Chen J, Gu D, Chen CS, Wu X, Hamm LL, Muntner P, Batuman V, Lee CH, Whelton PK $\mathrm{He} \mathrm{J}$. Association between the metabolic syndrome and chronic kidney disease in Chinese adults. Nephrol Dial Transplant 2007; 22: 1100-1106.

Supplementary Information accompanies the paper on Hypertension Research website (http://www.nature.com/hr) 